\title{
THE EVOLUTION OF RAPIDLY ROTATING B STARS \\ (Review Paper)
}

Robert Connon Smith

Astronomy Centre, University of Sussex, Falmer, Brighton BN1 9QH, U.K.

\begin{abstract}
Be stars are located in or near the main-sequence band for non-rotating stars. Although this stage of evolution is relatively well understood, there are two main effects that make it impossible to say whether all Be stars are in the same stage of evolution and, if so, what that stage is. One effect is the spread in observed magnitude and colour as a result of rotation. Correction for rotation is not possible because of the unknown inclination of any particular star's rotation axis to the line of sight and because it is not clear what the internal angular momentum distribution is or how it changes as a result of evolution. The other effect is that there are uncertainties in the theoretical evolutionary tracks because the amount of convective overshooting is unclear. Other mixing mechanisms that might in principle also confuse the tracks seem to be small near the main sequence. If Be stars are related to the $\beta$ Cephei and 53 Persei stars in the same part of the HR diagram, then the non-radial pulsation properties of Be stars may give a clue to their evolutionary state. The existence of a circumstellar disc or ring, however, tells us very little about the evolutionary stage of the underlying star. A useful way forward may be to try to understand individual stars in as much detail as possible.
\end{abstract}

\section{PREAMBLE}

This talk was originally to have been written by N. Baker and R. Kippenhahn, under the title "The Evolutionary State of the Be Stars". Unfortunately, Professor Kippenhahn, who was to have delivered the talk, was prevented from doing so by a slipped disc. I am grateful to Professors Baker and Kippenhahn for their abstract, which served as a starting point for my talk. However, any errors and omissions are my own, and I have changed the title to reflect a different emphasis. Because this talk was prepared at short notice, I make no claims for completeness in the list of references; I have tried to select 
representative recent articles and reviews, together with some important early papers.

\section{INTRODUCTION: BE STARS IN PERSPECTIVE}

The evolutionary state of Be stars is uncertain, and I am not going to provide a definitive statement. However, there are some things that we know from observation and it is worth summarising them. (Much fuller accounts of the observations are given by Abt and by Slettebak in earlier papers in this volume.) For the purposes of this paper, there are two properties of Be stars that are of particular importance. First of all, the luminosity classes fall in the range III - V and the spectral types in the range B0 - B9 (essentially by definition, although the 'Be phenomenon' may also ocur in some earlier and later types; see Slettebak, this volume). These facts tell us that to a first approximation we are dealing with stars that are on average above the zero age main sequence (ZAMS) but are in or near the main-sequence band and have masses in the range $4-20 M_{\odot}$. We are therefore concerned with evolution up to about the ignition of a $\mathrm{H}$-burning shell source, before the star begins to cross the Hertzsprung gap to the red giant region. Some of the uncertainties that afflict later stages of evolution, such as the role of semi-convection, are therefore not a serious problem for Be stars.

The second important observation is that the Doppler half-widths of the spectral lines are in the range $50-400 \mathrm{~km} \mathrm{~s}^{-1}$. Interpreting these half-widths in terms of rotation speeds, we can deduce that the class of Be stars contains the most rapidly rotating $B$ stars, at least some of which are rotating near, or possibly at, the critical velocity. However, many Be stars are rotating well below the critical velocity and there are certainly some normal $\mathrm{B}$ stars rotating faster than some $\mathrm{Be}$ stars. This tells us that rotation plays a role in the Be star phenomenon, but that it is not the only determining factor in whether a star shows the Be phenomenon.

Nonetheless, from the evolutionary point of view, the rotation must be taken into account and we have to try to answer two related questions:

How does very rapid rotation affect evolution near the main sequence?

How does evolution affect the observed rotation speed?

The rest of this talk will be a review of attempts to answer these questions, together with an acccount of the present uncertainties in evolutionary theory near the main sequence. I shall confine myself to a discussion of stars in the approximate mass range $4-20 M_{\odot}$, which have a convective core and a radiative envelope. Earlier reviews of rotation and evolution include Kraft (1970), Fricke \& Kippenhahn (1972), Tassoul (1978) and Moss \& Smith (1981), while general accounts of evolution near the main sequence have been given in many places. 
See, for example, the reviews by Iben (1967), Iben \& Renzini (1984), Maeder (1984) and the references therein.

\section{THE EFFECT OF EVOLUTION ON ROTATION}

The angular momentum distribution

If, as I have suggested in my earlier talk (this volume), shear instabilities dominate the redistribution of angular momentum inside stars, then we expect

$$
\Omega \simeq \Omega(P) \text { on the main sequence, }
$$

where $\Omega$ is the angular velocity and $P$ is the pressure, together with a mild variation with radius, fixed by the condition of marginal stability against a vertical shear. As a star evolves, its internal structure changes and mass shells move to a different radius. Because of conservation of angular momentum, this will in general destroy the original angular momentum distribution. Because the timescale for horizontal redistribution of angular momentum by turbulence is very short $\left(\simeq 10^{3} / \Omega\right.$, Zahn 1975$)$, there is likely to be virtually instantaneous readjustment of the horizontal $\Omega$ distribution so that $\Omega$ remains constant on pressure surfaces as the star evolves. Vertical redistribution is somewhat slower, with a timescale $\simeq 10^{3} /|d \Omega / d \ln r|($ Zahn 1974). However, that is still much faster than the typical timescale for evolution, which is the nuclear timescale except in the overall contraction phase after core hydrogen exhaustion. We therefore expect a rapid readjustment of the radial $\Omega$ distribution as well, but now with two constraints: not only must there be stability against vertical shear but there must be conservation of total angular momentum. (There will be a related constraint if the star is losing angular momentum via a stellar wind.)

This discussion has been for radiative zones, and has applied to the envelope of the evolving star. The angular momentum distribution in a convection zone is even less well understood. Two extreme assumptions for a convective core are:

uniform angular velocity throughout the core uniform angular momentum throughout the core (with a maximum value for $\Omega$ imposed near the rotation axis).

The first assumption is the commoner, although not for any strong theoretical reason (Tayler 1973). The question of whether or not the convective core exchanges angular momentum with the envelope during the star's evolution is important for the possible later development of a rapidly-spinning core, but is of little significance during main-sequence evolution and I shall not discuss it here. 


\section{Existing calculations}

There have not been many calculations of the effect of evolution on rotation and most of them have been discussed in the reviews by Fricke \& Kippenhahn (1972) and Moss \& Smith (1981). Essentially all of them make various simplifying assumptions about how the internal angular momentum redistribution occurs, without attempting to follow in detail the consequences of the many instabilities that actually determine the redistribution. In particular, there are no calculations for the case in which shear instabilities dominate, although the detailed calculations by Endal \& Sofia (1978) include the effect of shear instabilities.

The main assumptions that have been made can be summarised as follows:

1 No radial redistribution of angular momentum: local conservation of specific angular momentum $h\left(=\Omega \bar{\omega}^{2}\right.$, where $\bar{\omega}$ is distance from the rotation axis) on decoupled shells throughout radiative zones.

2 Complete redistribution: $\Omega$ uniform everywhere (rigid body rotation).

3 Partial redistribution:

a local conservation of $h$ in shells which have a stable molecular weight gradient; rigid body rotation in chemically homogeneous layers.

b detailed consideration of the effects of circulation and instabilities, allowing for finite redistribution timescales.

Examples of all these cases have been discussed by Moss \& Smith (1981). Cases 1 and 2 have been considered mainly as extreme examples (Oke \& Greenstein 1954), with the implication that the behaviour of real stars lies somewhere in between. Case 2 may be the closest to the case where shear instabilities dominate, especially if a magnetic field is also important. Case 3a represents an approximate attempt to allow for the stabilising influence of a $\mu$-gradient (Fricke \& Kippenhahn 1972). Observations of the range of rotational velocities for giants in the spectral type range B to G (Oke \& Greenstein 1954, Sandage 1955, Faber \& Danziger 1970) appear to exclude Case 1 but are compatible with Cases 2 and 3a. Bright giants and supergiants (classes II, Ib) may depart from this (Abt 1957, 1958) but these stars are too luminous to be relevant to Be stars.

Case 3b (Endal \& Sofia 1978) is the only attempt to treat the physics in detail. The series of papers by Endal \& Sofia $(1976,1978,1979)$ gives by far the most detailed discussion to date of how the angular momentum distribution changes with evolution and it forms the basis of the following discussion. I am not aware of any more recent calculations. The main drawback of their work is that they use diffusion equations to describe the effects of the circulation and instabilities that 
act on longer than dynamical timescales. This assumes that all these complicated motions tend to produce uniform rotation, which is by no means obvious (see my review in this volume).

\section{Evolution of $v_{\mathrm{eq}}$ in the main-sequence band}

The advantage of Cases 1 and 2 is that it is easy to predict, at least qualitatively, the results of more detailed calculations. For Case 1 (local conservation of angular momentum) we have in particular conservation of angular momentum for the shell at the surface and so we can write, for a star of mass $M$ and radius $R$,

$$
v_{\mathrm{eq}} R=\text { constant, or: } v_{\mathrm{eq}}=v_{\mathrm{ZAMS}} R_{\mathrm{ZAMS}}
$$

and

$$
\alpha=\frac{\Omega^{2} R^{3}}{G M}=\frac{v^{2} R}{G M}=\alpha_{\mathrm{ZAMS}} \frac{R_{\mathrm{ZAMS}}}{R} .
$$

Although we have not here taken into account the rotational distortion of the star, this predicts that both the equatorial rotation speed (and so the mean observed Doppler half-width) and the ratio of centrifugal force to gravity decrease monotonically as a result of evolution. This is hard to reconcile with observations of Be stars, which show no sign of a systematic decrease in Doppler half-width with distance above the main sequence (cf. Slettebak 1985, and discussion above).

In Case 2 (rigid body rotation at all times), we have to apply the constraint that the total angular momentum $J$ is constant:

$$
J=I \Omega\left(=M k^{2} R^{2} v / R\right)=\text { constant }
$$

Here we have written the moment of inertia $I$ in terms of the dimensionless radius of gyration $k$, which depends on the mass distribution within the star. In terms of $k$, we now have:

$$
v_{\mathrm{eq}}=v_{\mathrm{ZAMS}}\left(\frac{k_{\mathrm{ZAMS}}}{k}\right)^{2} \frac{R_{\mathrm{ZAMS}}}{R}
$$

and

$$
\alpha=\alpha_{\mathrm{ZAMS}}\left(\frac{k_{\mathrm{ZAMS}}}{k}\right)^{4} \frac{R_{\mathrm{ZAMS}}}{R} .
$$

If the mass distribution were unaltered by evolution, then the terms in $k$ would be unity and the results would be the same as for Case 1 . However, even in the main-sequence band, evolution is not homologous and stars begin to develop a core-halo structure, with the convective core actually decreasing in radius. This concentration of mass towards the centre causes $k$ to decrease during main-sequence evolution sufficiently rapidly to offset the increase in $R$ and so both $v_{\text {eq }}$ and $\alpha$ increase initially, especially $\alpha$, which can reach $\alpha_{\text {crit }}$ if the initial rotation 
speed is sufficiently large (Endal 1982). As was recognised already in the first calculations of this kind (Crampin \& Hoyle 1960), that may lead to mass loss. Although the mechanism of direct rotational mass loss is no longer regarded as the cause of the circumstellar shells in Be stars, the larger value of $\alpha$ may lead to an enhancement of an already-existing mass loss via a stellar wind.

At later stages of evolution, the changes in $k$ are much less and the increase in $R$ takes over, causing $v_{\text {eq }}$ and $\alpha$ to decrease again. However, that only occurs after $\mathrm{H}$-shell ignition and so probably beyond the Be star stage. Although I have quoted results for Case 2, the results for Case 3 b are qualitatively similar (Endal \& Sofia 1979, Figs. 5 and 6). Endal \& Sofia also point out an interesting feedback effect which enhances the initial increase in $v_{\text {eq }}$ and $\alpha$. This arises from the fact that on the ZAMS $k$ is smaller than for a non-rotating star of the same mass, because rotation causes the star to mimic a star of a smaller mass, which is more centrally concentrated. The increase in rotation of the core as a result of evolution thus leads to a further decrease in $k$, which in turn increases the rotation rate. This positive feedback is largest for those stars with the largest initial rotation rates and so these stars increase their rotation rates fastest (Endal 1982): a case of 'unto every one which hath shall be given' (Gospel according to St Luke, Ch.19, v.26).

The results of Case 2 calculations, and of the more detailed calculations of Endal \& Sofia $(1978,1979)$, are in reasonable accord with observed rotation speeds, although they do not explain the rapidly-rotating stars found near the main sequence in clusters (Slettebak 1985), which require a considerable spread of rotation speeds on the ZAMS as well as probably a range of evolutionary states.

\section{THE EFFECT OF ROTATION ON EVOLUTION}

Because rotation reduces the central temperature and luminosity of a star, it burns its available nuclear fuel more slowly, and so rotation causes a star to have a longer main-sequence lifetime. This can affect estimates of the ages of young clusters (see also below) but unless there is rotationally-induced mixing, discussed below, no more fuel is consumed and the shapes of the evolutionary tracks are hardly affected (Endal \& Sofia 1976, Fig. 2).

This statement immediately needs to be qualified: the tracks are unaffected when presented as total luminosity and spherically-averaged effective temperature. When aspect effects are taken into account, the tracks are considerably blurred, because stars with different orientations to the line of sight will be displaced in different directions and by different amounts in the HR diagram. The main effect (e.g. Collins \& Sonneborn 1977, Fig. 3) is to produce a broadening in $T_{\text {eff }}$ towards the red. This displacement to the right in the HR diagram simulates the effect of evolution, and may cause individual stars to appear more evolved than they are. 
The effect on cluster diagrams is to move the turn-off point to the red and so to make the clusters appear older than they are. For young clusters, this can have a much larger effect on age estimates than produced just by the lower total luminosity (Maeder 1971). Published calculations of the rotational displacements are for uniform or conservative rotation laws. Probably uniform rotation is the appropriate law (R.C. Smith, this volume), but it should be borne in mind that the real $\Omega$ distribution may be different and might give different results. However, it would be surprising if the differences were more than quantitative; the qualitative features are almost certainly correct. Another important qualitative effect of rotation is that the contracting core, if decoupled from the envelope, inevitably spins up and may end up rotating extremely rapidly (Kippenhahn et al. 1970, Endal \& Sofia 1976, 1978). Although these rapidly-spinning cores may have dramatic effects (Endal \& Sofia 1977) and may be relevant to the formation of pulsars, they do not develop until the red giant branch is reached and so are not relevant to the discussion of Be stars.

\section{OTHER EFFECTS THAT BLUR EVOLUTIONARY TRACKS}

Mass loss can have a major effect on evolutionary tracks, depending on the mass-loss rate. Tracks calculated by Sreenivasan \& Wilson (1978, Fig.1) show that for mass-loss rates of about $10^{-7} M_{\odot} \mathrm{y}^{-1}$ the effect is much larger than the rotational effects discussed in the last paragraph. However, although such a mass-loss rate may be appropriate for a normal B star, the observed mass-loss rates for Be stars are much lower, more like $10^{-9}$ to $10^{-10} M_{\odot} \mathrm{y}^{-1}$ (Vardya 1985). The blurring effect of mass loss on evolutionary tracks is therefore probably rather small for Be stars.

The most important effect is probably convective overshooting. It is widely recognised that the conventional mixing-length treatment of convection zones, in which all mixing is supposed to be confined to the regions with $\nabla>\nabla_{\mathrm{ad}}$, underestimates the actual size of the mixing zone, since the convective velocities do not actually go to zero where $\nabla=\nabla_{\text {add }}$. Estimates of the distance by which the convective motions overshoot the core vary considerably, depending on what non-local version of the mixing-length theory of convection is used (e.g. Maeder 1976, Bertelli et al. 1985, Doom 1985, Stothers \& Chin 1985), but the qualitative effect is clear: the extension of the convective core by overshooting beyond $\nabla=\nabla_{\text {ad }}$ leads to the mixing in of more fuel and so to the extension of the main-sequence lifetime and longer evolutionary tracks. Maeder \& Mermilliod (1981) find that lifetimes are increased by $20-40 \%$ for overshooting by about $15 \%$ of the mixing length. The resulting evolutionary tracks seem to account better for the spread in the observed colour-magnitude diagrams of clusters than do conventional calculations without overshooting (see Bertelli et al. 1985, Fig. 1). 
However, such comparisons between observation and theory are made for non-rotating models, whereas the observations include stars with a range of rotation speeds, so the deduced mixing by overshooting may be an overestimate. Also, these comparisons are statistical and do not help in deciding the evolutionary stage of a particular Be star.

It has been suggested by a number of authors (Maeder \& Mermilliod 1981, Sreenivasan \& Wilson 1985) that rotational mixing might also increase the effective core size and so lead to longer evolutionary tracks in the same way as does overshooting. If shear instabilities dominate the transport of angular momentum in rotating stellar interiors, then any mixing is likely to be largely horizontal (Zahn 1975) and slow Eddington-Sweet circulation will have little effect, especially if choked off by molecular weight gradients (Mestel 1953, Mestel \& Moss 1986). An apparently more plausible mechanism is proposed by Sreenivasan \& Wilson (1985), who note correctly that the effect of rotation is to flatten the pressure gradient by reducing the effective gravity. They then argue that the logarithmic radiative temperature gradient,

$$
\nabla_{\mathrm{rad}}=\frac{d \ln T}{d \ln P}=\frac{P d T / d r}{T d P / d r}
$$

is thereby increased, while the adiabatic gradient is unchanged at $\nabla_{\text {ad }}=(\gamma-1) / \gamma$. This would mean that more of the star had $\nabla_{\text {rad }}>\nabla_{\text {ad }}$ and so the convective core would be larger than in a non-rotating star. The effect of this would be similar to overshooting, and Sreenivasan \& Wilson found extensions of the core comparable to those found in most calculations of overshooting, giving main-sequence lifetimes longer than in the absence of rotation by as much as $35 \%$. However, their calculations are irrelevant, since their initial argument is wrong. Although it is true that the pressure gradient is flattened by rotation, the radiative temperature gradient is also flattened. Without a detailed model of a rotating star, it is therefore not possible to make a definite statement about how $\nabla_{\text {rad }}$ is affected by rotation. However, we know that rotation has the effect of making a star behave like a non-rotating star of a lower mass (Sackmann 1970) and so we expect that rotation will decrease the size of the convective core rather than increase it. That is confirmed by detailed calculations (e.g. Clement 1979, Fig. 2). I conclude that mixing induced by rotation can be ignored.

There are various other factors that introduce uncertainties into evolutionary calculations, such as semi-convection and opacities. Semi-convection has a large effect on evolutionary tracks during the core helium burning phase (e.g. Langer et al. 1985), but has only very small effects near the main sequence. A significant change in the accepted opacity tables could have an important effect anywhere in the HR diagram, but now that an outstanding discrepancy between the standard Cox-Stewart opacities and the newer Carson opacities has apparently been 
resolved (Carson et al 1984), there is no reason to suppose that there are any major uncertainties for calculations near the main sequence.

\section{OTHER CLUES TO THE EVOLUTIONARY STATE? Pulsation}

Despite the cautionary remarks by Balona, Bolton and others at this meeting, there does seem to be increasing evidence that many Be stars show non-radial pulsations (see reviews by Baade and by Percy in this volume, and by Smith, 1986). Although there is as yet no agreed excitation mechanism for these pulsations, it is possible that the existence or otherwise of non-radial pulsations is related to the evolutionary state of a star. It is therefore of interest to look for other stars in the same part of the HR diagram and see whether they also show evidence for pulsation. The best-known examples (Cox 1983) are the $\beta$ Cephei and 53 Persei stars. The $\beta$ Cephei, or $\beta$ Canis Majoris, variables show both radial and non-radial modes and include both fast and slow rotators. The 53 Persei variables show no evidence for radial modes but do display non-radial pulsations; they are all slow rotators.

Thus we have three groups of stars, all showing non-radial pulsations and in the same part of the HR diagram. There is no agreed excitation mechanism for any of these stars, despite many years of effort for the long-known $\beta$ Cep stars. A minimum hypothesis principle might suggest that the mechanisms should be at least related, if not the same, for the three groups of stars and that it would be helpful to think of all these stars as members of a single class of variable star. If so, and the pulsation is related to the evolutionary state, then we may get a clue to the evolutionary state of $\mathrm{Be}$ stars by looking at the other members of the class.

One of the complications with fitting evolutionary tracks to observations of $\mathrm{Be}$ stars is that they are rapidly rotating. That complication at least is not present for the slowly-rotating members of the $\beta$ Cep group and fits to evolutionary tracks for these stars suggest that they are in the late stages of hydrogen burning (Cox 1983, Fig. 1). Although this conclusion still suffers from uncertainties about the effects of overshooting, it is at least consistent with our ideas about Be stars.

Source of equatorial ring or disc

One feature that distinguishes Be stars from other B stars is the presence of an equatorial ring or disc. It was of course this that led Struve to his original rotational hypothesis, arguing that the ring was expelled by centrifugal forces. It is now recognised that this mechanism does not work; there is no net outward force on stellar material even when the surface is rotating at its critical velocity, and it is for that reason that Collins (this volume) has quite correctly 
attacked the use of the misleading expression 'break-up velocity'.

This recognition unfortunately removes one possible clue to the evolutionary state of Be stars. Early calculations for uniformly-rotating stars (e.g. Sackmann \& Anand 1970) assumed that mass loss occurred as soon as a star had evolved far enough (maintaining uniform rotation) for the surface to be rotating critically. This fixed the evolutionary state as being at a time during core hydrogen burning that depended only on the main-sequence rotation speed. Now that it is recognised that the cause of mass loss is radiation pressure or perhaps a centrifugal wind (Mestel 1968), rotation becomes only a contributory factor, by lowering the effective gravity and perhaps by providing an energy source for a turbulent energy flux. If non-radial pulsation occurs, that may also contribute to the mass loss, via a disc because non-radial modes have their largest amplitude in the equatorial plane (Penrod \& Smith 1985). With the additional freedom that the rotation of the core may evolve independently of the envelope, mass loss may now in principle occur almost anywhere on the evolutionary track, whatever the main-sequence rotation speed.

An alternative origin for the disc is accretion, rather than mass loss. The most natural way for this to happen would be by Roche lobe overflow from a companion (e.g. Harmanec 1982 and this volume), but one could also imagine that the disc could be a remnant either of a proto-planetary disc or at least of the process of star formation. If stars are formed in the very non-homologous way suggested by Larson (1969), with a central core forming first and then accreting the remains of the proto-stellar cloud, it would not be surprising if we observed some stars to be still 'not in utter nakedness, But trailing clouds of glory' (William Wordsworth, Ode, Intimations of Immortality, v). This reason for a circumstellar disc was proposed by Lynden-Bell \& Pringle (1974) for T Tauri stars, but there is no obvious reason why it should not also apply to more massive stars. If so, some Be stars (most likely the Herbig Ae/Be stars) could even be approaching the main sequence rather than leaving it. The main argument against that as a general explanation is the presence of Be stars in many clusters with well-defined main sequences that stretch to much lower masses (e.g. Slettebak 1985). Nonetheless, the observed disc could still be a remnant of the initial accretion process that formed the star. If so, it has been present since the pre-main-sequence stage and tells us nothing about the star's present state of evolution.

\section{CONCLUSIONS}

The best guess for how the evolution of a star affects its angular momentum distribution seems to be that uniform rotation of the radiative envelope is maintained as the star moves off the main sequence. 
The precise evolutionary state of Be stars is still uncertain, although there seems little doubt that they are in the main-sequence band. There are two main difficulties in comparing the observations with theoretical evolutionary tracks. The first is that the latter are blurred by a number of effects, principally uncertainties about the effects of overshooting, although there may also be some blurring as a result of a range of mass loss. Secondly, transformation from the observed magnitude and colour of a star to its theoretical luminosity and effective temperature is complicated by the effect of rotation. Correction for rotation is not unique (Collins \& Smith 1985) and the uncertainty in the inclination of a star's rotation axis to the line of sight adds a further blurring effect to the transformation.

All these effects make it very hard to pin down the evolutionary state of Be stars, especially as some are found very close to the ZAMS while others are considerably displaced (Slettebak 1985). An analogy with $\beta$ Cep stars suggests that the late main-sequence phase may be the best guess, but it is equally likely that stars showing the Be phenomenon occur throughout the main-sequence band. A second parameter (as well as age) may be the main-sequence value of $\alpha$, the ratio of centrifugal force to gravity, and it may well be that the Be phenomenon does not occur at a unique evolutionary stage but throughout the main-sequence band.

With so many possibly important parameters, the best approach may be to try to understand a few well-observed stars, by doing detailed evolutionary calculations incorporating observed values for the mass, rotation speed and mass loss rate. Hazlehurst \& Thomas (1970) have made calculations of this kind for stars in the Pleiades, but assumed that the Be stars were all rotating at their critical speeds. Revision of these calculations, and their extension to other clusters, could provide new insights into the evolutionary state of Be stars.

\section{Acknowledgement}

I am very grateful to Roger Discombe of the University of Sussex Computer Centre for his invaluable assistance in preparing the camera-ready text.

\section{REFERENCES}

Abt, H.A. (1957). Astrophys. J., 126, 503-8.

Abt, H.A. (1958). Astrophys. J., 127, 658-66.

Bertelli, G., Bressan, A.G. \& Chiosi, C. (1985). Astr. Astrophys., 150, 33-52.

Carson, T.R., Huebner, W.F., Magee, N.H. Jr \& Merts, A.L. (1984).

Astrophys. J., 283, 466-8.

Clement, M.J. (1979). Astrophys. J., 230, 230-42. 
Collins, G.W. II \& Sonneborn, G.H. (1977). Astrophys. J. Supp., 34, 41-94. Collins, G.W. II \& Smith, R.C. (1985). Mon. Not. R. astr. Soc., 213, 519-52. Cox, A.N. (1983). In Astrophysical Processes in Upper Main Sequence Stars, 13th Saas-Fee Course, eds. A. Maeder \& B. Hauck, pp. 1-165.

Sauverny: Geneva Observatory.

Crampin, J. \& Hoyle, F. (1960). Mon. Not. R. astr. Soc., 120, 33-42.

Doom, C. (1985). Astr. Astrophys., 142, 143-9.

Endal, A.S. (1982). In Be Stars, IAU Symp. No. 98, eds. M. Jaschek \&

H.-G. Groth, pp. 299-302. Dordrecht: D. Reidel.

Endal, A.S. \& Sofia, S. (1976). Astrophys. J., 210, 184-98.

Endal, A.S. \& Sofia, S. (1977). Phys. Rev. Lett., 399, 1429-32.

Endal, A.S. \& Sofia, S. (1978). Astrophys. J., 220, 279-90.

Endal, A.S. \& Sofia, S. (1979). Astrophys. J., 232, 531-40.

Faber, S.M. \& Danziger I.J. (1970). In Stellar Rotation, IAU Coll. No. 4, ed. A. Slettebak, pp. 39-46. Dordrecht: D. Reidel.

Fricke, K.J. \& Kippenhahn, R. (1972). Ann. Rev. Astr. Astrophys., 10, 45-72.

Harmanec, P. (1982). In Be Stars, IAU Symp. No. 98, eds. M. Jaschek \&

H.-G. Groth, pp. 279-97. Dordrecht: D. Reidel.

Hazlehurst, J. \& Thomas, H.-C. (1970). Mon. Not. R. astr. Soc., 150, 311-23.

Iben, I. Jr (1967). Ann. Rev. Astr. Astrophys., 5, 571-626.

Iben, I. Jr \& Renzini, A. (1984). Phys. Reports, 105, 329 406.

Kippenhahn, R., Meyer-Hofmeister, E. \& Thomas, H.-C. (1970).

Astr. Astrophys., $\underline{5}, 155-61$.

Kraft, R.P. (1970). In Spectroscopic Astrophysics, ed. G.H. Herbig, pp. 385-422.

Berkeley: University of California Press.

Langer, N., El Eid, M.F. \& Fricke, K.J. (1985). Astr. Astrophys., 145, 179-91.

Larson, R.B. (1969). Mon. Not. R. astr. Soc., 145, 271-95.

Lynden-Bell, D. \& Pringle, J.E. (1974). Mon. Not. R. astr. Soc., 168, 603-37.

Maeder, A. (1971). Astr. Astrophys., 10, 354-61.

Maeder, A. (1976). Astr. Astrophys., 47, 389-400.

Maeder, A. (1984). In Observational Tests of the Stellar Evolution Theory,

IAU Symp. No. 105, eds. A. Maeder \& A. Renzini, pp. 299-316.

Dordrecht: D. Reidel.

Maeder, A. \& Mermilliod, J.C. (1981). Astr. Astrophys., 93, 136-49.

Mestel, L. (1953). Mon. Not. R. astr. Soc., 113, 716-45.

Mestel, L. (1968). Mon. Not. R. astr. Soc., 138, 359-91.

Mestel, L. \& Moss, D. (1986). Mon. Not. R. astr. Soc., 221, 25-51.

Moss, D. \& Smith, R.C. (1981). Rep. Prog. Phys., 44, 831-91.

Oke, J.B. \& Greenstein, J.L. (1954). Astrophys. J., 120, 384-90.

Penrod, G.D. \& Smith, M.A. (1985). In The Origin of Non-Radiative

Heating/Momentum in Hot Stars, NASA Conf. Publ. CP-2358, eds.

A.B. Underhill \& A.G. Michalitsianos, pp. 53-6. Greenbelt: NASA. 
Sackmann, I.-J. (1970). Astr. Astrophys., $\underline{8}$, 76-84.

Sackmann, I.-J. \& Anand, S.P.S. (1970). Astrophys. J., 162, 105-24.

Sandage, A. (1955). Astrophys. J., 122, 263-70.

Slettebak, A. (1985). Astrophys. J. Supp., $\underline{59}$, 769-84.

Smith, M.A. (1986). In Proceedings, Unno Retirement Conference, ed. Y. Osaki, in press. Tokyo: University of Tokyo Press.

Sreenivasan, S.R. \& Wilson, W.J.F. (1978). Astr. Astrophys., 70, 755-61.

Sreenivasan, S.R. \& Wilson, W.J.F. (1985). Astrophys. J., 292, 506-10.

Stothers, R.B. \& Chin, C.-W. (1985). Astrophys. J., 292, 222-7.

Tassoul, J.-L. (1978). Theory of Rotating Stars. Princeton: Princeton University Press.

Tayler, R.J. (1973). Mon. Not. R. astr. Soc., 165, 39-52.

Vardya, M.S. (1985). Astrophys. J., 299, 255-64.

Zahn, J.-P. (1974). In Stellar Instability and Evolution, IAU Symp. No. 59, eds P. Ledoux, A. Noels \& A.W. Rodgers, pp. 185-95. Dordrecht: D. Reidel.

Zahn, J.-P. (1975) In Problems of Stellar Hydrodynamics, Liège Coll. No. 19, Mem. Soc. R. Sci. Liège, $6^{e}$ Sér., $\underline{8}, 31-4$. 


\section{DISCUSSION FOLLOWING R. SMITH}

Abt:

There are observational data on how rotational velocities change with evolution, done in the 1950's by Oke, Greenstein, Sandage, and Abt. They showed that the rotational change is between that of the solid-body rotation and conservation of angular momentum in shells.

Smith:

Yes. The most recent comparison with observational data is in the paper by Endal and Sofia (1979).

Garrison: stragglers.

If Be stars spend more time on the main sequence, there should be more Be blue

Smith:

I agree, but the effect is not large - only $10 \%$ or so compared to non-rotating stars, and in fact the non-Be stars are also rotating.

Collins: tion?

Is it your impression that rapid rotations are fully mixed throughout their evolu-

Smith:

No. Mixing is likely to be suppressed by molecular-weight gradients.

Cassinelli:

In regard to the work of Sreenivasan and Wilson, you stated that the adiabatic gradient also changes with rotation. Could you briefly explain that?

Castor:

Isn't the adiabatic gradient just a function of the radiation/gas pressure ratio? Is the change in the gradient a result of a change in this ratio?

Pesnell:

Do you need to include additional terms in the adiabatic gradient due to the presence of rotation?

Smith:

My explanation in the talk was a little misleading. What Sreenivasan and Wilson did was to recompute the adiabatic gradient by changing the pressure gradient to allow for rotational forces. However, they failed to allow for the fact that the temperature gradient is also flatter. I think this is the source of their error, rather than a neglect of the effect of rotation on the adiabatic gradient.

Friend:

MacGregor and Gilliland (1986) have re-done the analysis of Sreenivasan and Wilson (1985), taking into account the effect of rotation on both the radiative and adiabatic gradients. They find, as you suggest, that the core contracts rather than expands, contrary to the conclusion of Sreenivasan and Wilson. 